\title{
Producción científica y políticas de salud sobre el cigarro electrónico en Latinoamérica
}

\section{Scientific production and health policies on the electronic cigarette in Latin America}

\section{Sr. Editor:}

El cigarrillo electrónico (CE) es un dispositivo con forma similar al cigarro convencional, que libera determinadas dosis de nicotina a través de un proceso de calentamiento electrónico.

Desde su creación en el 2004, se ha utilizado como una alternativa al cigarro convencional, debido a diferentes argumentos que beneficiaban su consumo, aumentando de popularidad y logrando incluso que, los no fumadores lo incorporen en su uso diario (1), además, la falta de regulación sobre su venta permitió que la población joven y escolar puedan adquirirlo (2).

Esto generó que diversos países tomen decisiones políticas sobre el uso del CE, sin embargo, aun la evidencia científica podría ser insuficiente y necesitaría ser más confiable y significativa (3). Por lo tanto, una mayor contribución a partir de publicaciones científicas por cada país conllevaría a tomar decisiones más claras para su regulación, como sucedió con el cigarro convencional. Teniendo en cuenta ello, se realizó un análisis bibliométrico con el objetivo de evaluar y conocer la producción científica sobre el cigarro electrónico en Latinoamérica (LA).

Se realizó una búsqueda de la literatura en la base de datos de Scopus hasta septiembre del 2019 y se utilizó términos de encabezados de temas médicos (en inglés, MeSH) para «Electronic Nicotine Delivery Systems» y en países de LA. Se presentaron resultados de producción anual, tipo de publicación científica y tema de investigación.

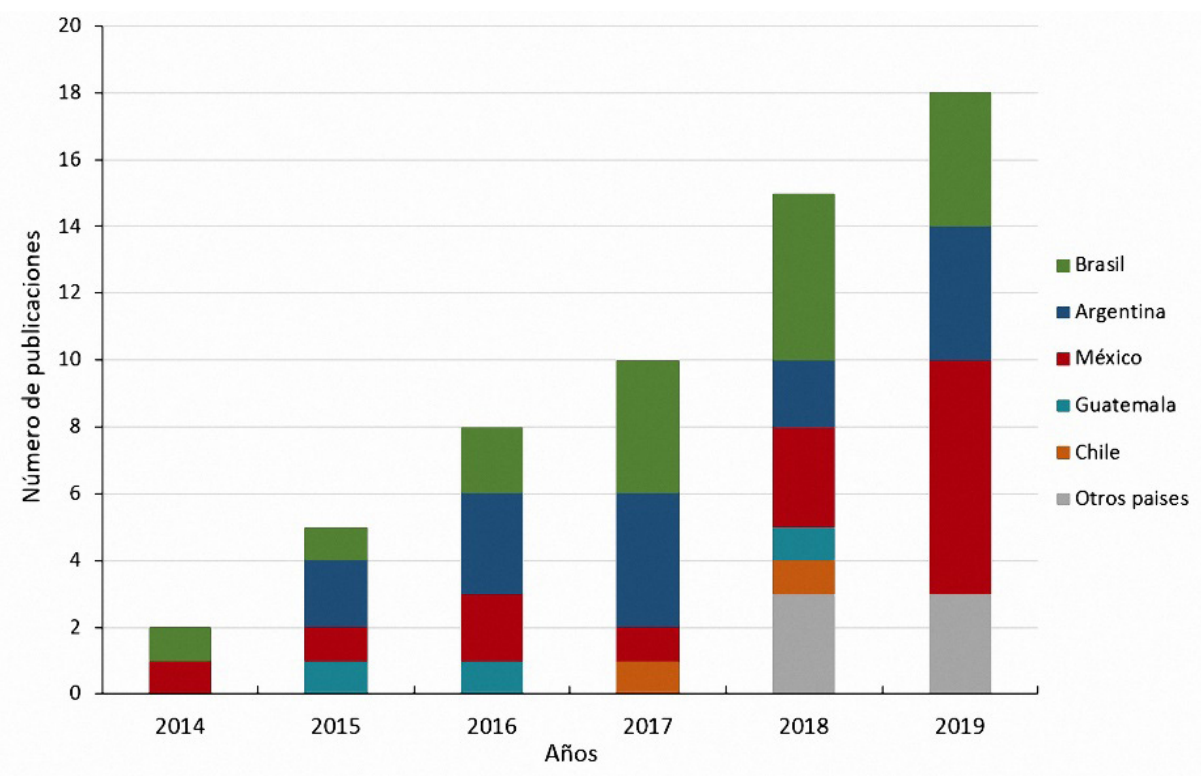

*Otros países: Colombia, Ecuador, Panamá, Puerto Rico, Uruguay.

Gráfico 1. Producción científica sobre el cigarro electrónico en países de Latinoamérica.

Asociación de Investigación y Ciencia de los Estudiantes de Medicina de la Universidad Privada San Juan Bautista. Ica, Perú. Unidad de Investigación en Bibliometría, Vicerrectorado de Investigación, Universidad San Ignacio de Loyola. Lima, Perú.

Estudiante de Medicina

Médico Cirujano 
Tabla 1. Características de los documentos publicados sobre el cigarro electrónico $(n=58)$.

\begin{tabular}{lcc}
\hline Características & $\mathbf{n}$ & $\mathbf{\%}$ \\
\hline Tipo de documento & & \\
Articulo original & 40 & 69,0 \\
Artículo de revisión & 10 & 17,2 \\
Carta & 4 & 7,0 \\
Editorial & 2 & 3,4 \\
Nota & 2 & 3,4 \\
Tema de investigación & & \\
Políticas sobre su consumo & 13 & 22,4 \\
Efectos en la salud & 15 & 25,9 \\
Epidemiología & 18 & 31,0 \\
Otros & 12 & 20,7 \\
\hline
\end{tabular}

Se encontró que, existen documentos publicados sobre el CE en países de LA a partir del 2014, con un total de 58 documentos, y que solo 10 países reportaron algún tipo de documento sobre el tema (principalmente, Brasil, México y Argentina); mientras que, en Perú y el resto de los países de LA, no se encontraron publicaciones sobre el tema (gráfico 1). Los documentos en mayor frecuencia fueron artículos originales $(n=40)$, mientras que, los temas que más se investigaron fueron con relación a epidemiología $(\mathrm{n}=18 ; 31 \%)$ y efectos en la salud $(15 ; 25,9 \%)$ (tabla $1)$.

Esta baja producción científica probablemente se deba a la ausencia de la participación de varios países de LA y explicaría la falta de políticas en salud que regulen el CE, pues de acuerdo con instituto Mundial para el Control del Tabaco, existen 98 países que tienen leyes sobre la regulación del CE (4), del cual, se identificaron 13 países de LA (Argentina, Brasil, Chile, Colombia, México, Costa Rica, Ecuador, Nicaragua, Panamá, Paraguay, Uruguay, Venezuela y El Salvador).

Por lo tanto, es necesario implementar estrategias que promuevan la investigación sobre el CE en LA, con orientación en publicación de artículos originales, pues son lo que contribuyen en gran porcentaje en la generación de nuevos conocimientos. Por último, en el Perú, a pesar de que se informó, que en mayo del 2019 cerca del $22 \%$ de los jóvenes están en uso del CE, aun el Ministerio de Salud se encuentra realizando propuestas para la prohibición de su comercialización (5). Esto podría darse de forma similar en otros países de nuestra región, permitiendo que puedan ser adquiridos en diferentes sitios web o centros comerciales sin ninguna restricción.
En conclusión, los autores consideramos que la investigación sobre el CE es escasa y es un tema nuevo para LA. Los países que no tuvieron publicaciones en revistas indizadas en Scopus, resulta importante desarrollar estudios clínico-epidemiológicos e incorporar marcos legales sobre la regulación del CE.

\section{Alex S. Huamán-Navarro ${ }^{1, a}$; Cristian Morán-Mariños ${ }^{2, b}$}

\section{Correspondencia:}

Cristian Andy Morán Mariños

Dirección: Jr. Pira 433 Urbanización el Parque

Naranjal. Lima, Perú.

Correo electrónico: cp.moran94@gmail.com

Teléfonos: +51931808126

\section{REFERENCIAS BIBLIOGRÁFICAS}

1. McMillen RC, Gottlieb MA, Shaefer RMW, Winick off JP, Klein JD. Trends in electronic cigarette use among U.S. Adults: Use is increasing in both smokers and nonsmokers. Nicotine Tob Res. 2015; 17(10):1195-202. Doi: https://doi.org/10.1093/ntr/ ntu213

2. Cullen KA, Ambrose BK, Gentzke AS, Apelberg BJ, Jamal A, King BA. Use of electronic cigarettes and any tobacco product among middle and high school students-United States, 2011-2018. Morb Mortal Wkly Rep. 2018; 67:1276-1277. Doi: http:// dx.doi.org/10.15585/mmwr.mm6722a3

3. Gaur S, Agnihotri R. Health effects of trace metals in electronic cigarette aerosols-a systematic review. Biol Trace Elem Res. 2019; 188(2):295-315. Doi: https://doi.org/10.1007/s12011-018-1423-x

4. Institute for Global Tobacco Control. Country Laws Regulating E-cigarettes: A Policy Scan. Baltimore, MD: Johns Hopkins Bloomberg School of Public Health. (Citado el 1 de diciembre del 2019) Dis ponible en: https://www.globaltobaccocontrol.org/ecigarette_policyscan

5. Ministerio de Salud. Perú: El cigarrillo electrónico incrementa cuatro veces más la adicción al tabaco convencional. Lima: Plataforma Digital única del Estado Peruano; 2019. (Citado el 15 de octubre de 2019) Disponible en: https://www.gob.pe/institucion/ minsa/noticias/28917-el-cigarrillo-electronicoincrementa-cuatroveces-mas-la-adiccion-al-tabacoconvenciona

Recibido: 29/11/2019

Aceptado: 30/12/2020 